\title{
New Complex Hyperbolic Function Solutions for the (2+1)-Dimensional Dispersive Long Water-Wave System
}

\author{
Hasan Bulut ${ }^{1, *,+}$ and Haci Mehmet Baskonus ${ }^{2,+}$ \\ 1 Department of Mathematics, Firat University, Elazig 23119, Turkey \\ 2 Department of Computer Engineering, Tunceli University, Tunceli 62100, Turkey; hmbaskonus@gmail.com \\ * Correspondence: hbulut@firat.edu.tr; Tel.: +90-424-237-0000 \\ + These authors contributed equally to this work.
}

Academic Editor: Mehmet Pakdemirli

Received: 18 February 2016; Accepted: 18 March 2016; Published: 24 March 2016

\begin{abstract}
In this paper, new algorithms called the "Modified exp $(-\Omega)$-expansion function method" and "Improved Bernoulli sub-equation function method" have been proposed. The first algorithm is based on the $\exp (-\Omega(\xi))$-expansion method; the latter is based on the Bernoulli sub-Ordinary Differential Equation method. The methods proposed have been expressed comprehensively in this manuscript. The analytical solutions and application results are presented by drawing the two- and three-dimensional surfaces of solutions such as hyperbolic, complex, trigonometric and exponential solutions for the (2+1)-dimensional dispersive long water-wave system. Finally, a conclusion has been presented by mentioning the important discoveries in this manuscript.
\end{abstract}

Keywords: modified $\exp (-\Omega(\xi))$-expansion function method; improved Bernoulli sub-equation function method; the (2+1)-dimensional dispersive long water-wave system; hyperbolic function solution; trigonometric function solution; complex function solution; exponential function solution

\section{Introduction}

In recent years, studies on the nonlinear differential equations (NDEs) and systems (NDESs) have become a significant field among scientists. This is why many engineering problems can be represented by using NDEs and with the rapid development of computational algorithms. Therefore, many mathematical models have been proposed to understand of such problems in scientific and engineering such as the optical fiber communications, coastal and oceans engineering, fluid dynamics, plasma physics, chemical physics and many other scientific applications. Yong-Sik Cho, Dae-Hee Sohn and Seung Oh Lee have conducted a study on shallow-water equations for distant propagation of tsunamis [1]. Furthermore, even some important diseases such as dengue epidemics, tuberculosis, HIV, AIDS, tsunamis, malaria, and cholera have been investigated by many scientists from all over the word [2-7]. One of them is to obtain various solutions of coastal, oceans and fluid problems such as approximate, numerical, analytical and traveling wave solutions. Some important traveling wave solutions for nonlinear differential equations have been investigated by different authors [8-15]. In the rest of this manuscript; we have explained the fundamental properties of the modified $\exp (-\Omega(\xi))$-expansion function method (MEFM) and Improved Bernoulli sub-equation function method (IBSEFM) in Section 2. We have studied to obtain some new analytical solutions such as hyperbolic, exponential, and complex hyperbolic function solutions by applying MEFM and IBSEFM to the (2+1)-dimensional dispersive long water-wave (DLW) system defined by [16]:

$$
\begin{gathered}
u_{y t}+u_{x x y}-2 v_{x x}-\left(u^{2}\right)_{x y}=0 \\
v_{t}-v_{x x}-2(u v)_{x}=0
\end{gathered}
$$


where $u=u(x, y, t)$ represents the horizontal velocity of water and $v=v(x, y, t)$ gives the deviation height from the equilibrium position of the liquid [16]. The solutions of (1) are very helpful for coastal scientists and engineers to apply the nonlinear water model to coastal and harbor design [17-19]. Equation (1) was used to model nonlinear and dispersive long gravity waves traveling in two horizontal directions on shallow waters of uniform depth. Equation (1) appears in many scientific applications such as nonlinear fiber optics, plasma physics, fluid dynamics, and coastal engineering [16].

\section{Fundamental Properties of Methods}

\subsection{The Modified $\operatorname{Exp}(-\Omega(\xi))$-Expansion Function Method}

Let us consider the following nonlinear partial differential equations systems for functions $u, v[20,21]:$

$$
\begin{aligned}
& P_{1}\left(u_{x}, v_{x}, u_{t}, v_{t}, u_{y}, v_{y}, \cdots\right)=0, \\
& P_{2}\left(u_{x}, v_{x}, u_{t}, v_{t}, u_{y}, v_{y}, \cdots\right)=0,
\end{aligned}
$$

where $u=u(x, y, t), v=v(x, y, t)$ are unknown functions, and $P i(i=0,1)$ are polynomials in $u=u(x, y, t)$, $v=v(x, y, t)$

Step 1: Combine the real variables $x, y$ and $t$ with a compound variable $\xi$ :

$$
\begin{gathered}
u(x, y, t)=U(\xi), \xi=k x+w y-c t \\
v(x, y, t)=V(\xi), \xi=k x+w y-c t \\
u_{x}=\frac{\partial u}{\partial x}=k U^{\prime}(\xi), u_{y}=\frac{\partial u}{\partial y}=w U^{\prime}(\xi), u_{t}=\frac{\partial u}{\partial t}=-c U^{\prime}(\xi), \\
v_{x}=\frac{\partial v}{\partial x}=k V^{\prime}(\xi), v_{y}=\frac{\partial v}{\partial y}=w V^{\prime}(\xi), v_{t}=\frac{\partial v}{\partial t}=-c V^{\prime}(\xi),
\end{gathered}
$$

where $\omega, c, k$ are both constants and non-zero. By travelling wave transformation, Equation (2) under the terms of Equations (3) and (4) transforms Equation (2) system in a nonlinear ordinary differential equation (NODE) as the following:

$$
\operatorname{NODE}\left(U, U^{\prime}, U^{\prime \prime}, U^{\prime \prime \prime}, \cdots\right)=0,
$$

where NODE is a polynomial of $U$ and $U^{\prime}=\frac{d U}{d \xi}, U^{\prime \prime}=\frac{d^{2} U}{d \xi^{2}}, U^{\prime \prime \prime}=\frac{d^{3} U}{d \xi^{3}}$.

Step 2: Conjecture of the travelling wave solutions for Equation (5) can be stated in the following form:

$$
U(\xi)=\frac{\sum_{i=0}^{N} A_{i}\left[\exp (-\Omega(\xi))^{i}\right.}{\sum_{j=0}^{M} B_{j}[\exp (-\Omega(\xi))]^{j}}=\frac{A_{0}+A_{1} \exp (-\Omega(\xi))+\cdots+A_{N} \exp (-N \Omega(\xi))}{B_{0}+B_{1} \exp (-\Omega(\xi))+\cdots+B_{M} \exp (-M \Omega(\xi))^{2}}
$$

where $A_{i}(0 \leqslant \mathrm{i} \leqslant N)$ and $B_{j}(0 \leqslant j \leqslant M)$ are constants to be determined, such that $A_{N} \neq 0, B_{M} \neq 0$, and $\Omega=\Omega(\xi)$ satisfies the following ordinary differential equation:

$$
\Omega^{\prime}=\mu \exp (\Omega)+\exp (-\Omega)+\lambda .
$$

The following exact analytical solutions can be written from Equation (7) [22-24]: 
Family-1: If $\mu \neq 0, \lambda^{2}-4 \mu>0$,

$$
\Omega(\xi)=\ln \left(\frac{-\sqrt{\lambda^{2}-4 \mu}}{2 \mu} \tanh \left(\frac{\sqrt{\lambda^{2}-4 \mu}}{2}(\xi+\varepsilon)\right)-\frac{\lambda}{2 \mu}\right) .
$$

Family-2: When $\mu \neq 0, \lambda^{2}-4 \mu<0$,

$$
\Omega(\xi)=\ln \left(\frac{\sqrt{-\lambda^{2}+4 \mu}}{2 \mu} \tan \left(\frac{\sqrt{-\lambda^{2}+4 \mu}}{2}(\xi+\varepsilon)\right)-\frac{\lambda}{2 \mu}\right) .
$$

Family-3: When $\mu=0, \lambda \neq 0$, and $\lambda^{2}-4 \mu>0$,

$$
\Omega(\xi)=-\ln \left(\frac{\lambda}{\exp (\lambda(\xi+\varepsilon))-1}\right)
$$

Family-4: When $\mu \neq 0, \lambda \neq 0$, and $\lambda^{2}-4 \mu=0$,

$$
\Omega(\xi)=\ln \left(-\frac{2 \lambda(\xi+\varepsilon)+4}{\lambda^{2}(\xi+\varepsilon)}\right)
$$

Family-5: When $\mu=0, \lambda=0$, and $\lambda^{2}-4 \mu=0$,

$$
\Omega(\xi)=\ln (\xi+\varepsilon),
$$

where $A_{0}, A_{1}, \ldots, A_{N}, B_{0}, B_{1}, \ldots, B_{M}, \varepsilon, \lambda, \mu$ are real constants to be identified later. $\varepsilon$ is constant of integration. The positive integer $N$ and $M$ can be identified by taking the balance rule between the highest order derivatives and the nonlinear terms occurring in Equation (5).

Step 3: According to different values of $N$ and $M$, we can find various forms of Equation (6). By putting Equations (6) in (5), we can obtain a polynomial of $\exp (-\Omega(\xi))$. If we equal to zero all the coefficients of same power of $\exp (-\Omega(\xi))$, we can obtain a system of equations. When we solve this system with the aid of Wolfram Mathematica 9, this process gives different values of coefficients $A_{0}, A_{1}, \ldots, A_{N}$, and $B_{0}, B_{1}, \ldots, B_{M}, \varepsilon$. If we put these coefficients in Equation (6) by considering family conditions, we obtain many analytical solutions for Equation (2).

\subsection{Improved Bernoulli Sub-Equation Function Method (IBSEFM)}

IBSEFM formed by improving the Bernoulli sub-equation function method [25] will be given in this sub-section. Therefore, we consider the following steps.

Step 1. Let us consider the nonlinear partial differential equations system as the following:

$$
\begin{aligned}
& P_{1}\left(u_{x}, v_{x}, u_{t}, v_{t}, u_{y}, v_{y}, \cdots\right)=0, \\
& P_{2}\left(u_{x}, v_{x}, u_{t}, v_{t}, u_{y}, v_{y}, \cdots\right)=0,
\end{aligned}
$$

and take the wave transformation

$$
\begin{aligned}
& u(x, y, t)=U(\xi), \xi=k x+w y-c t, \\
& v(x, y, t)=V(\xi), \xi=k x+w y-c t,
\end{aligned}
$$

where $w, k, c$ are both constants and non-zero and will be determined later. Substituting Equations (14) into (13) converts a nonlinear ordinary differential equation (NODE) as the following:

$$
\operatorname{NODE}\left(U, U^{\prime}, U^{\prime \prime}, U^{\prime \prime \prime}, \cdots\right)=0 \text {, }
$$


where NODE is a polynomial of $U$, and, $U^{\prime}=\frac{d U}{d \xi^{\prime}}, U^{\prime \prime}=\frac{d^{2} U}{d \xi^{2}}, U^{\prime \prime \prime}=\frac{d^{3} U}{d \xi^{3}}$.

Step 2. Considering the trial equation of the solution in Equation (15), it can be written as the following:

$$
U=U(\xi)=\frac{\sum_{i=0}^{n} a_{i} F^{i}}{\sum_{j=0}^{m} b_{j} F^{j}}=\frac{a_{0}+a_{1} F+a_{2} F^{2}+\cdots+a_{n} F^{n}}{b_{0}+b_{1} F+b_{2} F^{2}+\cdots+b_{m} F^{m}},
$$

and, according to the Bernoulli theory,

$$
F^{\prime}=b F+d F^{M}, b \neq 0, d \neq 0, M \in R-\{0,1,2\},
$$

where $F=F(\xi)$ is Bernoulli differential polynomial and $F^{\prime}=\frac{d F}{d \xi}=\frac{d F(\xi)}{d \xi}$. Substituting the above relations in Equation (15) yields an equation of polynomial $\Omega(F)$ of $F=F(\xi)$

$$
\Omega(F)=\rho_{s} F^{S}+\cdots+\rho_{1} F+\rho_{0}=0 .
$$

According to the balance principle, we can get some values of $n, m$ and $M$.

Step 3. Letting the coefficients of $\Omega(F)$ all be zero will yield an algebraic equations system

$$
\rho_{i}=0, i=0, \cdots, s .
$$

Solving this system, we will determine the values of $k, w, c, a_{0}, a_{1}, a_{2}, \ldots a_{n}, b_{0}, b_{1}, b_{2}, \ldots, b_{m}$.

Step 4. When we solve nonlinear Bernoulli differential Equation (17), we obtain, according to $b$ and $d$, two situations as the following:

$$
\begin{gathered}
F(\xi)=\left[\frac{-d}{b}+\frac{\varepsilon}{e^{b(M-1) \xi}}\right]^{\frac{1}{1-M}}, b \neq d, \\
F(\xi)=\left[\frac{(\varepsilon-1)+(\varepsilon+1) \tanh \left(\frac{b(1-M) \xi}{2}\right)}{1-\tanh \left(\frac{b(1-M) \xi}{2}\right)}\right]^{\frac{1}{1-M}}, b=d, \varepsilon \in \mathbb{R},
\end{gathered}
$$

where $\varepsilon$ is both an integration constant and non-zero. Using a complete discrimination system for polynomial of $F(\xi)$, we solve Equation (16) with the help of using Wolfram Mathematica 9 and classify the exact solutions to Equation (16). For a better interpretations of results obtained in this way, we plot two- and three-dimensional surfaces of the solutions obtained by taking suitable parameters.

\section{The Implementations of Techniques}

In this subsection of the paper, we have obtained new analytical solutions to the DLW system (1) by using MEFM and IBSEFM.

\subsection{Application of MEFM}

Let us consider the travelling wave solutions of the DLW system (1) and we perform the transformation as the following;

$$
\begin{aligned}
& u(x, y, t)=U(\xi), \xi=k x+w y-c t \\
& v(x, y, t)=V(\xi), \xi=k x+w y-c t .
\end{aligned}
$$


First of all, if we consider the following transformations for the DLW system (1),

$$
v(x, y, t)=u_{y}(x, y, t)=\frac{\partial u(x, y, t)}{\partial y},
$$

we can obtain following partial differential equations:

$$
\begin{gathered}
u_{y t}+u_{x x y}-2 u_{y x x}-\left(u^{2}\right)_{x y}=0, \\
u_{y t}-u_{y x x}-2\left(u u_{y}\right)_{x}=0 .
\end{gathered}
$$

When we use the travelling wave transformations Equation (22),

$$
\begin{gathered}
u_{y t}=-c w U^{\prime \prime}, u_{x x y}=w k^{2} U^{\prime \prime \prime}, u_{y x x}=w k^{2} U^{\prime \prime \prime}, u_{x y}=w k U^{\prime \prime}, u_{x}=k U^{\prime}, u_{y}=w U^{\prime} \\
\left(u^{2}\right)_{x y}=2\left(u u_{x}\right)_{y}=2\left(u_{y} u_{x}+u u_{x y}\right)=2 w k\left(U U^{\prime}\right)^{\prime}
\end{gathered}
$$

and using Equations (26) in (24), we obtain the following NODE;

$$
-c U^{\prime \prime}-k^{2} U^{\prime \prime \prime}-2 k\left(U U^{\prime}\right)^{\prime}=0 .
$$

If we integrate Equation (27) twice by getting zero the integration constants, we obtain the following NODE

$$
-c U-k^{2} U^{\prime}-k U^{2}=0 .
$$

When we use Equations (26) in (25), after integrating twice by getting zero the integration constants we obtain the same NODE as Equation (28) below:

$$
-c U-k^{2} U^{\prime}-k U^{2}=0 .
$$

When we rearrange to Equation (6), with the help of balance principle between $U^{\prime}$ and $U^{2}$, we obtain the term for suitability:

$$
N=M+1
$$

This relationship gives rise to various analytical solutions to the DLW system (1) as the following:

\section{Case-1}

If we choose $M=1$ and $N=2$, we can write the following equations for Equation (6):

$$
U=\frac{A_{0}+A_{1} \exp (-\Omega)+A_{2} \exp (-2 \Omega)}{B_{0}+B_{1} \exp (-\Omega)}=\frac{\Upsilon}{\Psi},
$$

and

$$
U^{\prime}=\frac{\Upsilon^{\prime} \Psi-\Psi^{\prime} \Upsilon}{\Psi^{2}},
$$

where $A_{2} \neq 0, B_{1} \neq 0$ and $\Omega=\Omega(\xi)$. When we use Equations $(31,32)$ in the $(29)$, we get a system of equations for Equation (29) from the coefficients of polynomial of $\exp (-\Omega(\xi))$. Solving this system with the help of Wolfram Mathematica 9 yields the following coefficients:

\section{Case 1.1:}

$$
A_{1}=\frac{2 A_{0}}{\lambda}+k B_{0}, A_{2}=\frac{2 k B_{0}}{\lambda}, B_{1}=\frac{2 B_{0}}{\lambda}, \mu=\frac{A_{0}\left(-A_{0}+k \lambda B_{0}\right)}{k^{2} B_{0}^{2}}, c=k\left(k \lambda-\frac{2 A_{0}}{B_{0}}\right) .
$$




\section{Case 1.2:}

$$
\begin{gathered}
A_{0}=\frac{A_{1}^{2}}{4 A_{2}}, B_{0}=\frac{-i \lambda \sqrt{A_{2}\left(A_{1}-\lambda A_{2}\right)}}{\sqrt{2 c}}, B_{1}=\frac{-i \sqrt{2 A_{2}\left(A_{1}-\lambda A_{2}\right)}}{\sqrt{c}}, k=\frac{i \sqrt{c A_{2}}}{\sqrt{2 A_{1}-2 \lambda A_{2}}}, \\
\mu=\frac{-A_{1}\left(A_{1}-2 \lambda A_{2}\right)}{4 A_{2}^{2}} .
\end{gathered}
$$

\section{Case 1.3:}

$$
\begin{gathered}
A_{0}=\frac{-\lambda}{4}\left(-2 A_{1}+\lambda A_{2}\right), B_{0}=\frac{-i \lambda \sqrt{A_{2}\left(A_{1}-\lambda A_{2}\right)}}{\sqrt{2 c}}, B_{1}=\frac{-i \sqrt{2 A_{2}\left(A_{1}-\lambda A_{2}\right)}}{\sqrt{c}}, \\
k=\frac{i \sqrt{c A_{2}}}{\sqrt{2 A_{1}-2 \lambda A_{2}}}, \mu=-\frac{3 \lambda^{2}}{4}-\frac{A_{1}\left(A_{1}-2 \lambda A_{2}\right)}{A_{2}^{2}} .
\end{gathered}
$$

Case 1.4:

$$
\begin{gathered}
A_{0}=\frac{A_{1}^{2}}{4 A_{2}}, B_{0}=\frac{i \lambda \sqrt{A_{2}\left(A_{1}-\lambda A_{2}\right)}}{\sqrt{2 c}}, B_{1}=\frac{i \sqrt{2 A_{2}\left(A_{1}-\lambda A_{2}\right)}}{\sqrt{c}}, k=\frac{-i \sqrt{c A_{2}}}{\sqrt{2 A_{1}-2 \lambda A_{2}}}, \\
\mu=-\frac{A_{1}\left(A_{1}-2 \lambda A_{2}\right)}{4 A_{2}^{2}} .
\end{gathered}
$$

Case 1.5:

$$
A_{0}=\frac{k^{2} B_{0}^{2}}{4 A_{2}}, A_{1}=k B_{0}, B_{1}=\frac{A_{2}}{k}, \mu=\frac{3 k^{2} B_{0}^{2}}{4 A_{2}^{2}}, \lambda=\frac{2 k B_{0}}{A_{2}}, c=\frac{2 k^{3} B_{0}}{A_{2}} .
$$

Therefore, when we substitute coefficients Equations (33) along with (8) in (31) for $u(x, y, t)$ and in Equation (23) for $v(x, y, t)$, we can obtain hyperbolic function solution to the DLW system (1) as the following, under the condition of Family- $1 ; \lambda^{2}-4 \mu=\left(-2 A_{0}+k \lambda B_{0}\right)^{2} / k^{2} B_{0}^{2}>0$ :

$$
\begin{gathered}
u_{1}(x, y, t)=\frac{A_{0}}{B_{0}}+\frac{2 A_{0}\left(A_{0}-k \lambda B_{0}\right)}{k \lambda B_{0}^{2}+2 k \beta B_{0}^{2} \tanh (\beta f(x, y, t))} \\
v_{1}(x, y, t)=\frac{w A_{0}\left(-2 A_{0}+k \lambda B_{0}\right)^{2}\left(-A_{0}+k \lambda B_{0}\right) \operatorname{sech}^{2}(\beta f(x, y, t))}{k B_{0}^{2}+\left[k \lambda B_{0}+\left(-2 A_{0}+k \lambda B_{0}\right) \tanh ^{2}(\beta f(x, y, t))\right]}
\end{gathered}
$$

where $f(x, y, t)=\varepsilon+w y+k(x-\lambda k t)+\frac{2 k A_{0}}{B_{0}} t, \beta=\frac{-2 A_{0}+k \lambda B_{0}}{2 k B_{0}}$, and, $A_{0}, B_{0}, \lambda, k, w, \varepsilon$ are both constants and non-zero.

For Equation (34), if we use coefficients Equations (34) along with (8) in (31) for $u(x, y, t)$ and in Equation (23) for $v(x, y, t)$, we can obtain new complex hyperbolic function solution to the DLW system(1) as the following, under the condition of Family- $1 ; \lambda^{2}-4 \mu=\left(A_{1}-\lambda A_{2}\right)^{2} / A_{2}^{2}>0$ :

$$
\begin{aligned}
u_{2}(x, y, t) & =\frac{i A_{1}^{2} \sqrt{c \omega}[1+\tanh [\beta f(x, y, t)]]^{2}}{2 \sqrt{2 A_{2}}\left[A_{1}+\lambda A_{2}[-1+\tanh (\beta f(x, y, t))]\left[-\lambda A_{2}-\omega \tanh (\beta f(x, y, t))\right]\right.}, \\
v_{2}(x, y, t) & =\frac{\gamma(x, y, t) A_{1}^{2} \omega^{3 / 2}(-\omega)(1+\sigma(x, y, t))^{2}}{4 \sqrt{2} A_{2}^{3 / 2} \ell(x, y, t) g(x, y, t)^{2}}-\frac{\gamma(x, y, t) A_{1}^{2} \omega^{3 / 2}(1+\sigma(x, y, t))}{2 \sqrt{2} A_{2}^{3 / 2} \ell(x, y, t) g(x, y, t)} \\
& +\frac{\gamma(x, y, t) A_{1}^{2} \omega^{3 / 2}(1+\sigma(x, y, t))^{2}}{4 \sqrt{2} \sqrt{A_{2}} \ell(x, y, t) g(x, y, t)},
\end{aligned}
$$

where $\beta=\frac{A_{1}-\lambda A_{2}}{2 A_{2}}, f(x, y, t)=\varepsilon-c t+w y+\frac{i \sqrt{c A_{2}} x}{\sqrt{2\left(A_{1}-\lambda A_{2}\right)}}, \omega=A_{1}-\lambda A_{2}$, $\gamma(x, y, t)=i \sqrt{c} w \operatorname{Sech}\left[\frac{\omega f(x, y, t)}{2 A_{2}}\right]^{2}, \sigma(x, y, t)=\tanh \left[\frac{\omega f(x, y, t)}{2 A_{2}}\right], \ell(x, y, t)=A_{1}+$ $\lambda A_{2}(-1+\sigma(x, y, t)), g(x, y, t)=-\lambda A_{2}-\omega \sigma(x, y, t)$ and, $A_{0}, B_{0}, \lambda, k, w, \varepsilon$ are constants and not zero. 
Considering Equations (35) along with (8) in (31) for $u(x, y, t)$ and in Equation (23) for $v(x, y, t)$, we can obtain another new complex hyperbolic function solution to the DLW system (1) as the following, under the condition of Family- $1 ; \lambda^{2}-4 \mu=4\left(A_{1}-\lambda A_{2}\right)^{2} / A_{2}^{2}>0$ :

$$
\begin{gathered}
u_{3}(x, y, t)=\frac{i \sqrt{c \omega}\left(2 A_{1}-\lambda A_{2}\right)(1+\tanh [\omega f(x, y, t)])}{\sqrt{2} A_{2}^{3 / 2}\left(\lambda+\frac{2 \omega}{A_{2}} \tanh [\omega f(x, y, t)]\right)}, \\
v_{3}(x, y, t)=\frac{-i \sqrt{c} \omega\left(2 A_{1}-3 \lambda A_{2}\right) \omega^{3 / 2}\left(2 A_{1}-\lambda A_{2}\right)}{\sqrt{2} A_{2}^{3 / 2}\left(\lambda A_{2} \operatorname{Cosh}\left[\frac{\omega}{A_{2}} f(x, y, t)\right]+2 \omega \operatorname{Sinh}\left[\frac{\omega}{A_{2}} f(x, y, t)\right]\right)^{2}},
\end{gathered}
$$

being $f(x, y, t)=\varepsilon-c t+w y+\frac{i \sqrt{c A_{2}} x}{\sqrt{2 \omega}}, \omega=A_{1}-\lambda A_{2}$.

Substituting Equations (36) along with (8) in (31) for $u(x, y, t)$ and in Equation (23) for $v(x, y, t)$, we can obtain another new complex hyperbolic function solution to the DLW system (1) as the following, under the condition of Family-1; $\lambda^{2}-4 \mu=\left(A_{1}-\lambda A_{2}\right)^{2} / A_{2}^{2}>0$ :

$$
\begin{gathered}
u_{4}(x, y, t)=\frac{i \sqrt{c \omega} A_{1}^{2}(1+\sigma(x, y, t))^{2}}{2 \sqrt{2 A_{2}\left(A_{1}+\lambda A_{2}(-1+\sigma(x, y, t))\right)}\left(-\lambda A_{2}-\omega \sigma(x, y, t)\right)^{2}}, \\
v_{4}(x, y, t)=\frac{\gamma(x, y, t) A_{1}^{2} \omega^{5 / 2}(1+\sigma(x, y, t))^{2}}{4 \sqrt{2} A_{2}^{3 / 2} \ell(x, y, t)\left(g(x, y, t)^{2}\right)}+\frac{\gamma(x, y, t) A_{1}^{2} \omega^{3 / 2}(1+\sigma(x, y, t))}{2 \sqrt{2} A_{2}^{3 / 2} \ell(x, y, t) g(x, y, t)} \\
-\frac{\gamma(x, y, t) A_{1}^{2} \omega^{3 / 2}(1+\sigma(x, y, t))^{2}}{4 \sqrt{2 A_{2}} \ell^{2}(x, y, t) g(x, y, t)},
\end{gathered}
$$

where $f(x, y, t)=\varepsilon-c t+w y-\frac{i \sqrt{c A_{2}}}{\sqrt{2 \omega}} x, \gamma(x, y, t)=i \sqrt{c} w \operatorname{Sech}^{2}\left[\frac{\omega}{2 A_{2}} f(x, y, t)\right], \sigma(x, y, t)=$ $\tanh \left[\frac{\omega}{2 A_{2}} f(x, y, t)\right], \omega=A_{1}-\lambda A_{2}, \ell(x, y, t)=A_{1}+\lambda A_{2}(-1+\sigma(x, y, t)), g(x, y, t)=-\lambda A_{2}-$ $\omega \sigma(x, y, t)$.

Taking Equations (37) along with (8) in (31) for $u(x, y, t)$ and in Equation (23) for $v(x, y, t)$, we can obtain another new hyperbolic function solution to the DLW system(1) as the following, under the condition of Family- $1 ; \lambda^{2}-4 \mu=k^{2} B_{0}^{2} / A_{2}^{2}>0$ :

$$
\begin{gathered}
u_{5}(x, y, t)=\frac{k^{2} B_{0}}{4 A_{2}}\left(1-\frac{6}{2+\tanh [h(x, y, t)]}+\frac{3}{1+2 \tanh [h(x, y, t)]}\right), \\
v_{5}(x, y, t)=-\frac{9 k^{3} w \operatorname{sech}^{4}[h(x, y, t)] B_{0}^{2}}{4 A_{2}^{2}(2+\tanh [h(x, y, t)])^{2}(1+2 \tanh [h(x, y, t)])^{2}},
\end{gathered}
$$

where $h(x, y, t)=\frac{k B_{0}}{2 A_{2}^{2}}\left((\varepsilon+k x+w y) A_{2}-2 k^{3} B_{0} t\right)$.

\section{Case-2}

If we choose $M=2$ and $N=3$, we can write following equations for Equation (6):

$$
U=\frac{A_{0}+A_{1} \exp (-\Omega)+A_{2} \exp (-2 \Omega)+A_{3} \exp (-3 \Omega)}{B_{0}+B_{1} \exp (-\Omega)+B_{2} \exp (-2 \Omega)}=\frac{\Upsilon}{\Psi},
$$

and

$$
U^{\prime}=\frac{\Upsilon^{\prime} \Psi-\Psi^{\prime} \Upsilon}{\Psi^{2}}
$$


where $A_{3} \neq 0, B_{2} \neq 0$ and $\Omega=\Omega(\xi)$. When we use Equations (43) and (44) in the (29), we get a system of equations for Equation (29) from the coefficients of polynomial of $\exp (-\Omega(\xi))$. Solving this system with the help of Wolfram Mathematica 9 yields the following coefficients:

\section{Case 2.1:}

$$
\begin{gathered}
A_{0}=\frac{\left(\lambda-\sqrt{\lambda^{2}-4 \mu}\right) A_{3} B_{0}}{2 B_{2}}, A_{1}=\frac{A_{3}\left(2 B_{0}+\left(\lambda-\sqrt{\lambda^{2}-4 \mu}\right) B_{1}\right)}{2 B_{2}}, \\
A_{2}=\frac{1}{2} A_{3}\left(\lambda-\sqrt{\lambda^{2}-4 \mu}+\frac{2 B_{1}}{B_{2}}\right), c=\frac{\sqrt{\lambda^{2}-4 \mu} A_{3}^{2}}{B_{2}^{2}}, k=\frac{A_{3}}{B_{2}},
\end{gathered}
$$

Case 2.2:

$$
\begin{gathered}
A_{3}=\frac{1}{2 \mu^{3}}\left[\left(\lambda^{3}+\lambda^{2} \sqrt{p}-3 \lambda \mu-\sqrt{p} \mu\right) A_{0}+\mu\left(-(\lambda r-2 \mu) A_{1}+r \mu A_{2}\right)\right], \\
B_{0}=\frac{r p^{1 / 4} A_{0}}{2 \sqrt{c} \mu}, B_{1}=\frac{p^{1 / 4}\left(-(\lambda r-2 \mu) A_{0}+r \mu A_{1}\right)}{2 \sqrt{c} \mu^{2}}, \\
B_{2}=\frac{p^{1 / 4}\left(\lambda^{3}+\lambda^{2} \sqrt{p}-3 \lambda \mu-\sqrt{p} \mu\right) A_{0}+\mu\left(-(\lambda r-2 \mu) A_{1}+r \mu A_{2}\right)}{A_{0}+\mu\left(-(\lambda r-2 \mu) A_{1}+r \mu A_{2}\right)}, \\
k=\frac{\sqrt{c}}{p^{1 / 4}}, p=\lambda^{2}-4 \mu, r=\lambda+\sqrt{p} .
\end{gathered}
$$

When considering Equations (45) along with (8) in (31) for $u(x, y, t)$ and in Equation (23) for $v(x, y, t)$, another new hyperbolic function solution to the DLW system(1) can be obtained as the following, under the condition of Family- $1 ; \lambda^{2}-4 \mu>0$ :

$$
\begin{gathered}
u_{6}(x, y, t)=\frac{A_{3}}{2 B_{2}}\left(\lambda-\sqrt{\psi}-\frac{4 \mu}{\lambda+\sqrt{\psi} \tanh \left[\frac{1}{2} \sqrt{\psi}(z(x, y, t))\right]}\right) \\
v_{6}(x, y, t)=\frac{w \psi \mu A_{3}}{\left(\lambda \operatorname{Cosh}\left[\frac{1}{2} \sqrt{\psi}(z(x, y, t))\right]+\sqrt{\psi} \operatorname{Sinh}\left[\frac{1}{2} \sqrt{\psi}(z(x, y, t))\right]\right)^{2}},
\end{gathered}
$$

where $z(x, y, t)=\varepsilon+w y+\frac{A_{3}}{B_{2}^{2}}\left(-t \sqrt{\lambda^{2}-4 \mu} A_{3}+x B_{2}\right)$, and $\psi=\lambda^{2}-4 \mu$.

Putting Equation (46) along with Equation (8) in Equation (31) for $u(x, y, t)$ and in Equation (23) for $v(x, y, t)$ gives another new hyperbolic function solution to the DLW system (1) as the following, under the condition of Family-1; $\lambda^{2}-4 \mu>0$ :

$$
\begin{gathered}
u_{7}(x, y, t)=\frac{\sqrt{c} \psi^{1 / 4}\left(\lambda^{2}-\lambda \sqrt{\psi}+2\left(-1+e^{-(\theta(x, y, t))}\right) \mu\right) \operatorname{sech}^{2}\left[\frac{1}{2}(\theta(x, y, t))\right]}{-8 \mu-2 \psi \operatorname{sech}^{2}\left[\frac{1}{2}(\theta(x, y, t))\right]}, \\
v_{7}(x, y, t)=\frac{\sqrt{c} w \psi^{1 / 4} \mu\left(2 \sqrt{\psi} \mu+\sqrt{\psi}\left(\lambda^{2}-2 \mu\right) \cosh [\theta(x, y, t)]-\lambda \psi \sinh [\theta(x, y, t)]\right)}{\left(\lambda^{2}-2 \mu+2 \mu \cosh [\theta(x, y, t)]\right)^{2}},
\end{gathered}
$$

where $\theta(x, y, t)=\sqrt{\psi}\left(\varepsilon-c t+w y+\frac{\sqrt{c} x}{\psi^{1 / 4}}\right), \quad \psi=\lambda^{2}-4 \mu$.

\subsection{Application of IBSEFM}

Let us consider the travelling wave solutions of the DLW system (1), and we perform the transformation as the following:

$$
\begin{aligned}
& u(x, y, t)=U(\xi), \xi=k x+w y-c t \\
& v(x, y, t)=V(\xi), \xi=k x+w y-c t .
\end{aligned}
$$


First of all, if we consider the above transformations for the DLW system (1), we can obtain the same NODE as Equation (29):

$$
-c U-k^{2} U^{\prime}-k U^{2}=0 .
$$

When we rearrange to Equation (16), with the help of balance principle between $U^{\prime}$ and $U^{2}$, we obtain the term for suitability:

$$
m+M=n+1
$$

This relationship allows us various analytical solution forms for the DLW system (1).

\section{Case-1}

If we choose $M=n=3$ and $m=1$, we can write the following equations for Equation (6):

$$
U(\xi)=\frac{a_{0}+a_{1} F+a_{2} F^{2}+a_{3} F^{3}}{b_{0}+b_{1} F}=\frac{\Upsilon}{\Psi}
$$

and

$$
U^{\prime}=\frac{\Upsilon^{\prime} \Psi-\Psi^{\prime} \Upsilon}{\Psi^{2}},
$$

where $a_{3} \neq 0, b_{1} \neq 0$ and $F=F(\xi)$.When we use Equations (52) and (53) in Equation (50), we get a system of equations for Equation (50) from the coefficients of polynomial of $F$. Solving this system with the help of Wolfram Mathematica 9 yields the following coefficients:

\section{Case 1.1:}

$$
a_{0}=0, a_{1}=0, a_{2}=\frac{i \sqrt{2} \sqrt{c} d b_{0}}{\sqrt{b}}, a_{3}=\frac{i \sqrt{2} \sqrt{c} d b_{1}}{\sqrt{b}}, k=-\frac{i \sqrt{c}}{\sqrt{2} \sqrt{b}}
$$

Case 1.2:

$$
a_{0}=\sqrt{2} \sqrt{b} \sqrt{c} b_{0}, a_{1}=\sqrt{2} \sqrt{b} \sqrt{c} b_{1}, a_{2}=\frac{\sqrt{2} \sqrt{c} d b_{0}}{\sqrt{b}}, a_{3}=\frac{\sqrt{2} \sqrt{c} d b_{1}}{\sqrt{b}}, k=-\frac{\sqrt{c}}{\sqrt{2} \sqrt{b}}
$$

Case 1.3:

$$
a_{0}=0, a_{1}=0, a_{2}=0, a_{3}=-\frac{i \sqrt{2} \sqrt{c} d b_{1}}{\sqrt{b}}, b_{0}=0, k=\frac{i \sqrt{c}}{\sqrt{2} \sqrt{b}} .
$$

If Equations (54) along with (8) is put into (51) for $u(x, y, t)$ and in Equation (23) for $v(x, y, t)$, new complex exponential function solution to the DLW system(1) can be obtained as the following:

$$
\begin{gathered}
u_{8}(x, y, t)=\frac{i \sqrt{2} \sqrt{c} d}{\sqrt{b}\left(-\frac{d}{b}+e^{\left.2 b c t+i \sqrt{2} \sqrt{b} \sqrt{c} x-2 b w y_{\mathcal{E}}\right)}\right.} \\
v_{8}(x, y, t)=\frac{2 i \sqrt{2} b^{5 / 2} \sqrt{c} d e^{2 b c t+i \sqrt{2} \sqrt{b} \sqrt{c} x-2 b w y_{\varepsilon} w}}{\left(d e^{2 b w y}-b e^{2 b c t+i \sqrt{2} \sqrt{b} \sqrt{c} x} \mathcal{E}\right)^{2}} .
\end{gathered}
$$

When we use coefficients Equations (55) along with (8) in (51) for $u(x, y, t)$ and in Equation (23) for $v(x, y, t)$, we can obtain another new exponential function solution to the DLW system (1) as the following:

$$
\begin{gathered}
u_{9}(x, y, t)=\frac{\sqrt{2} b^{3 / 2} \sqrt{c} \varepsilon}{-d e^{-2 b c t-\sqrt{2} \sqrt{b} \sqrt{c} x+2 b w y}+b \varepsilon}, \\
v_{9}(x, y, t)=\frac{2 \sqrt{2} b^{5 / 2} \sqrt{c} d e^{2 b c t+\sqrt{2} \sqrt{b} \sqrt{c} x+2 b w y} \varepsilon w}{\left(d e^{2 b w y}-b e^{2 b c t+\sqrt{2} \sqrt{b} \sqrt{c} x} \varepsilon\right)^{2}} .
\end{gathered}
$$


Substituting Equations (56) along with (8) in (51) for $u(x, y, t)$ and in Equation (23) for $v(x, y, t)$, we can obtain another new complex exponential function solution to the DLW system(1) as the following:

$$
\begin{gathered}
u_{10}(x, y, t)=-\frac{i \sqrt{2} \sqrt{c} d}{\sqrt{b}\left(-\frac{d}{b}+e^{2 b c t-i \sqrt{2} \sqrt{b} \sqrt{c} x-2 b w y} \varepsilon\right)}, \\
v_{10}(x, y, t)=-\frac{2 i \sqrt{2} b^{5 / 2} \sqrt{c} d e^{2 b c t+i \sqrt{2} \sqrt{b} \sqrt{c} x-2 b w y} \varepsilon w}{\left(d e^{i \sqrt{2} \sqrt{b} \sqrt{c} x+2 b w y}-b e^{2 b c t} \mathcal{E}\right)^{2}} .
\end{gathered}
$$

\section{Conclusions}

The MEFM and IBSEFM have been applied to the (2+1)-dimensional dispersive long water-wave system Equation (1). Then, these methods which are newly submitted to literature in this paper have given new complex and exponential functional solutions such as Equations (39)-(41) and (57)-(59) for the DLW system Equation (1). We have shown that these analytical solutions are verified in Equation (1). Furthermore, we have already found the similar hyperbolic function solutions such as Equations (38), (42), (47), (48), and (58) with analytical solutions obtained by Wazwaz [16]. Moreover, complex function solutions such as Equations (39)-(41), (57), and (59) obtained in this paper by MEFM and IBSEFM are new analytical solutions when compared with analytical solutions obtained by Wazwaz [16]. These results are very helpful for coastal and civil engineers to apply the nonlinear water model to coastal and harbor design [16]. Figures 1-15 have been plotted by using Wolfram Mathematica 9 under the suitable values of parameters. It can be predicted that they are very useful for applications in engineering and science, especially, coastal and ocean engineering. To the best of our knowledge, applications of MEFM and IBSEFM to the DLW system (1) have not previously been submitted to literature. We think that these newly modified methods can also be applied to other models that result from engineering, science, coastal and ocean engineering.
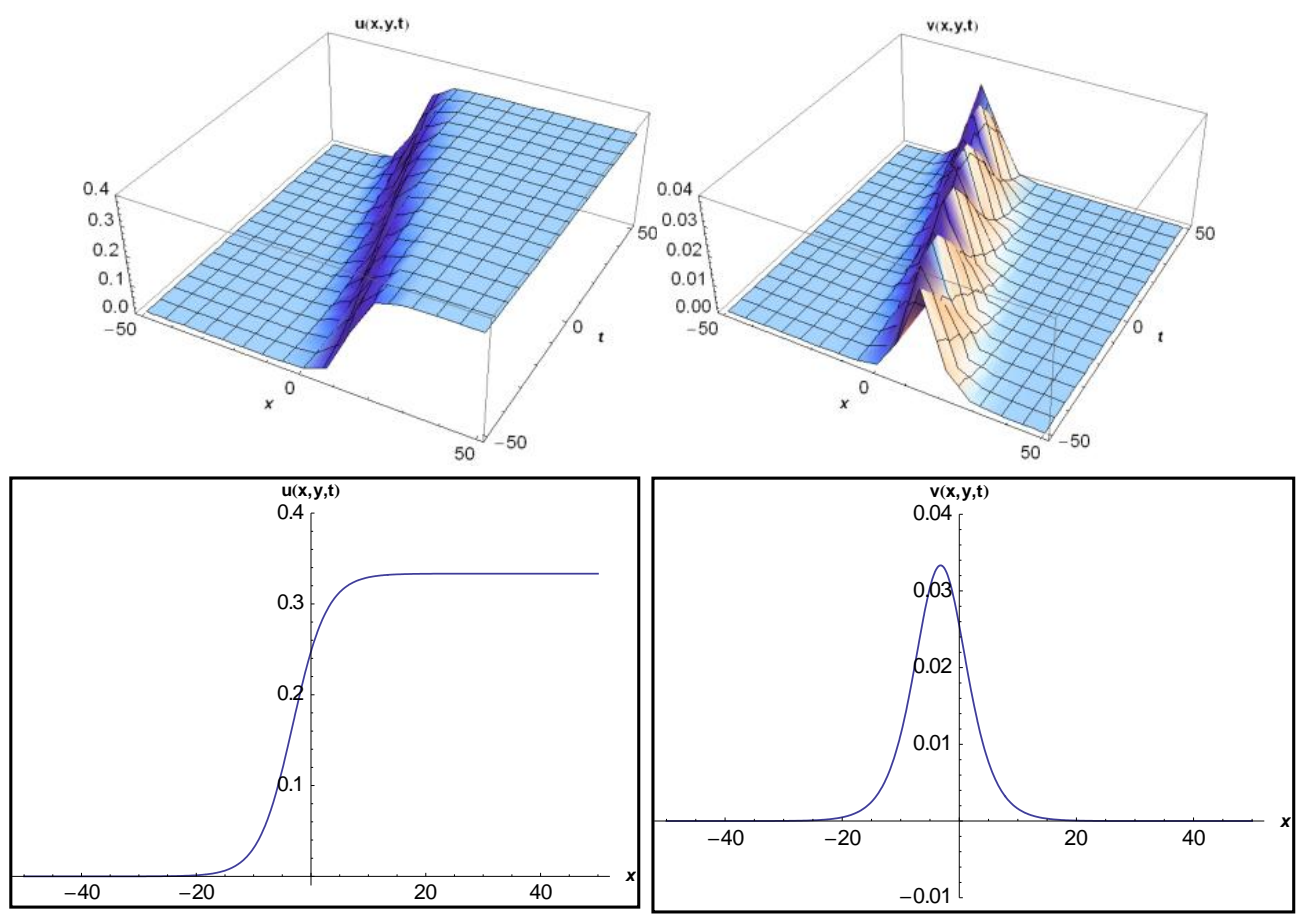

Figure 1. The $3 \mathrm{D}$ and $2 \mathrm{D}$ surfaces of Equation (38) by considering the values $A_{0}=2, B_{0}=3, k=5$, $\lambda=0.2, \omega=6, \varepsilon=4, y=0.2,-50<x<50,-50<t<50$, and $t=0.2$, for $2 \mathrm{D}$ surfaces. 

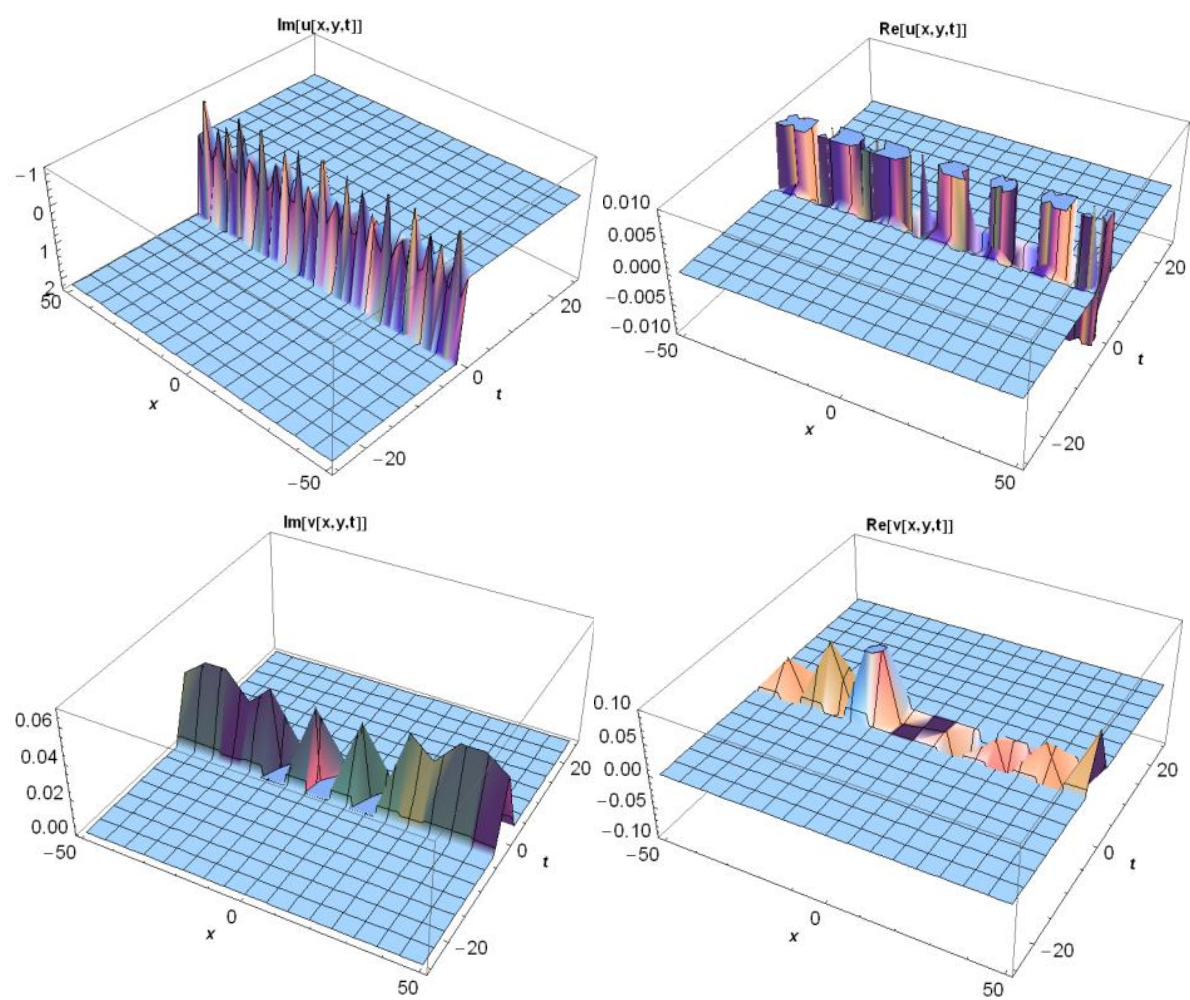

Figure 2. The 3D surfaces of Equation (39) by considering the values $A_{1}=2, A_{2}=3, c=5, \lambda=0.2$, $w=0.1, \varepsilon=-3, y=0.2,-50<x<50,-25<t<25$.
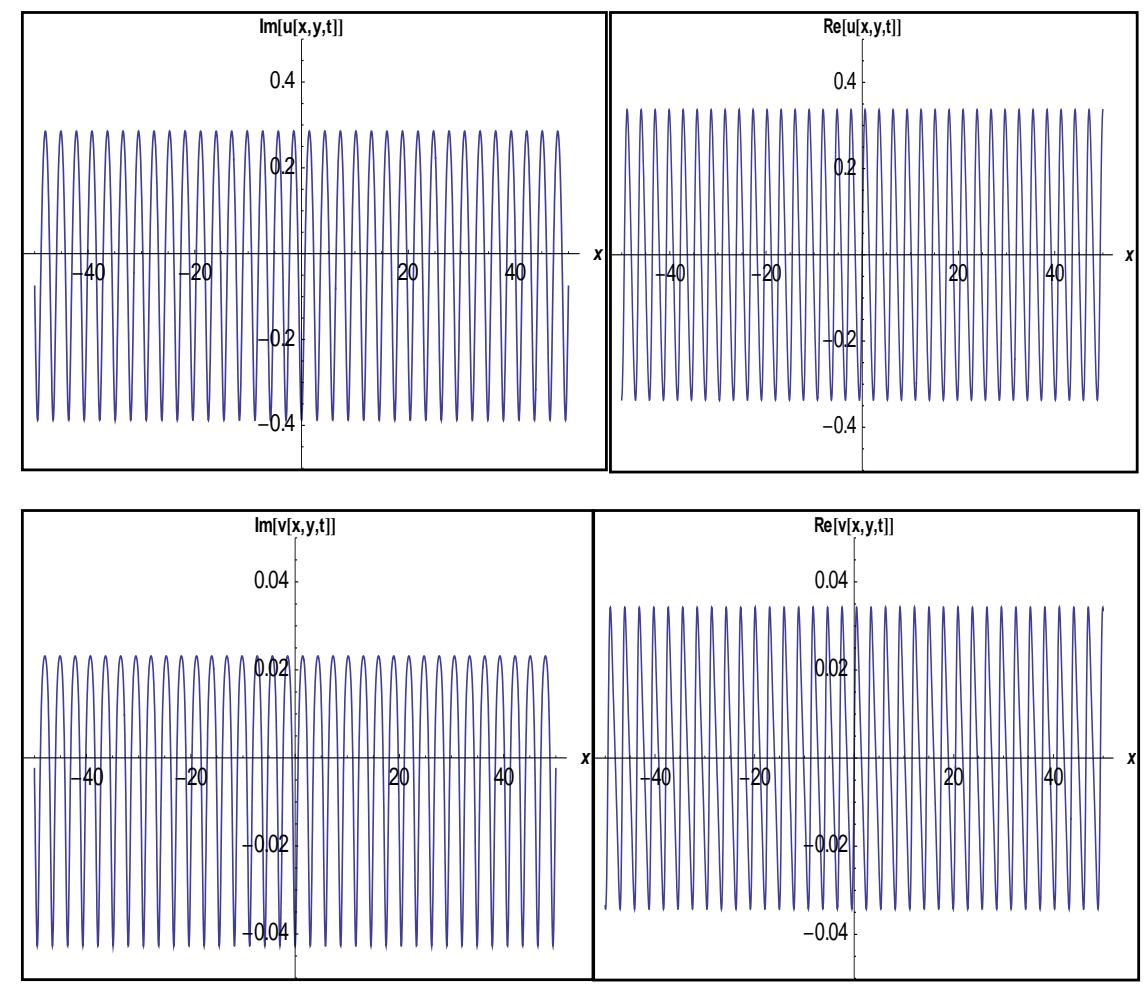

Figure 3. The 2D surfaces of Equation (39) by considering the values $A_{1}=2, A_{2}=3, c=5, \lambda=0.2$, $\omega=0.1, \varepsilon=-3, y=t=0.2,-50<x<50$. 

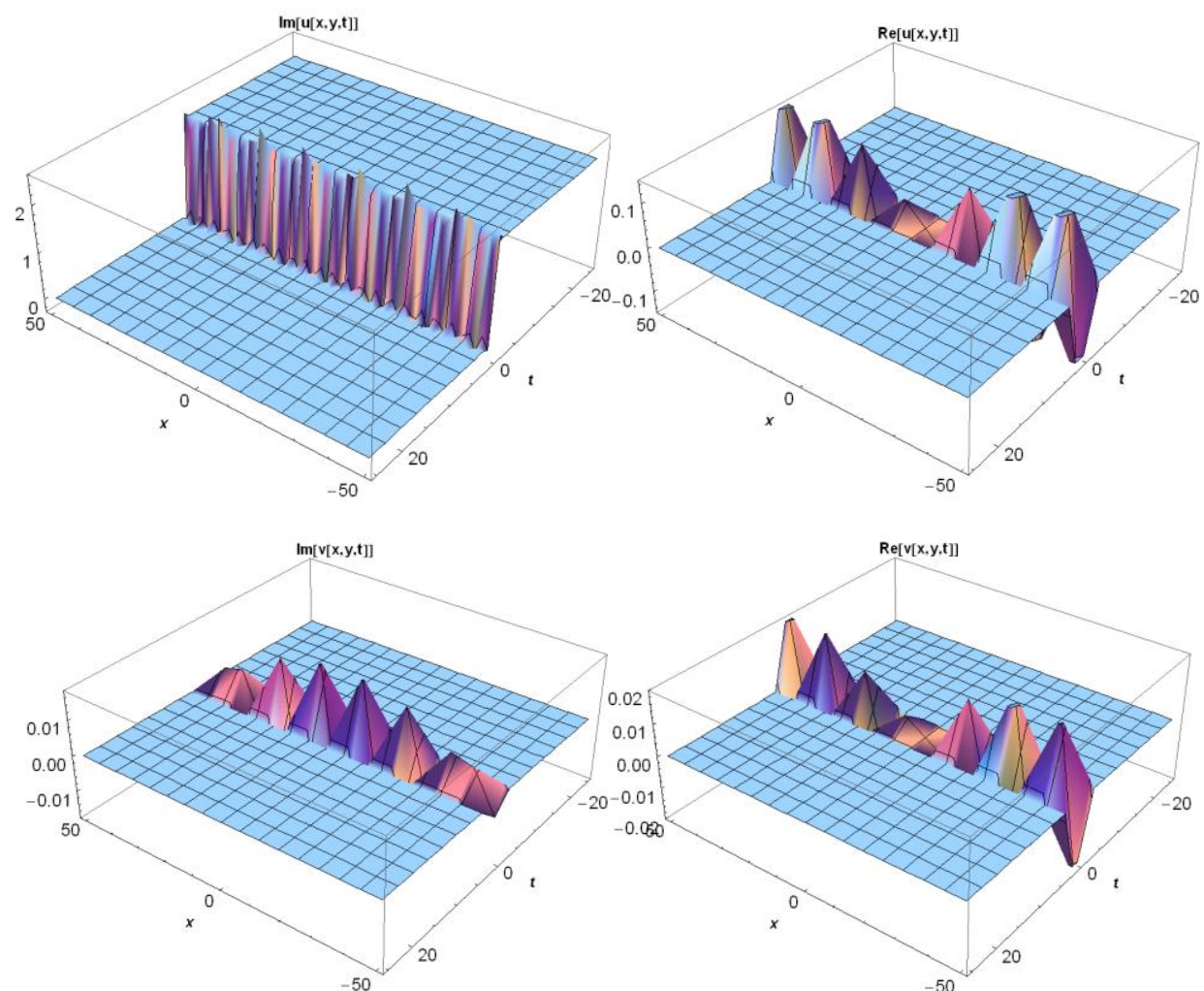

Figure 4. The 3D surfaces of Equation (40) by considering the values $A_{1}=2, A_{2}=3, c=5, \lambda=0.2$, $\omega=0.12, \varepsilon=-3, y=0.2,-50<x<50,-25<t<25$.
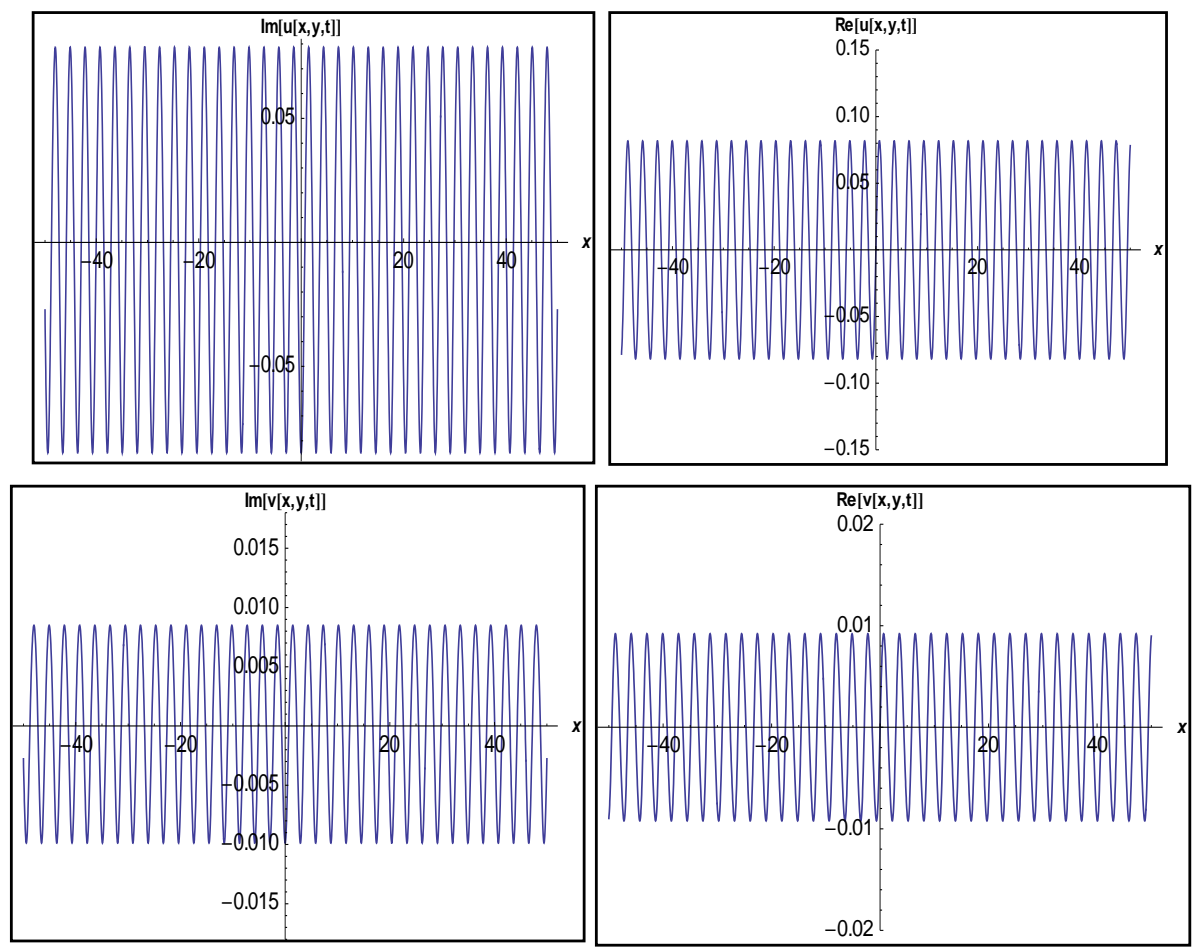

Figure 5. The $2 \mathrm{D}$ surfaces of Equation (40) by considering the values $A_{1}=2, A_{2}=3, c=5$, $\lambda=0.2, w=0.12, \varepsilon=-3, y=t=0.2,-50<x<50$. 

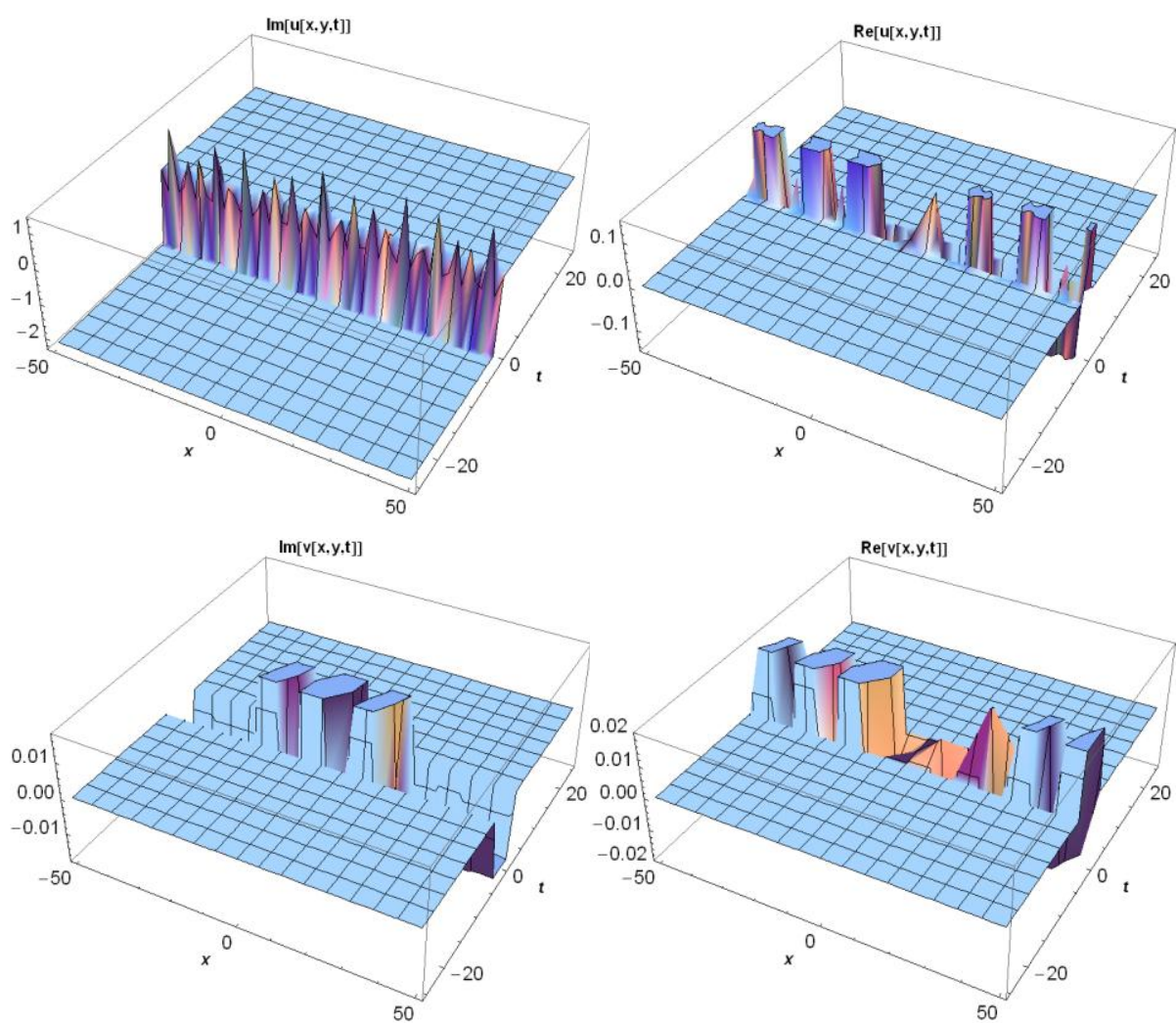

Figure 6. The $3 \mathrm{D}$ surfaces of Equation (41) by considering the values $A_{1}=2, A_{2}=3, c=5$, $\lambda=0.2, w=0.12, \varepsilon=-3, y=0.2,-50<x<50,-25<t<25$.
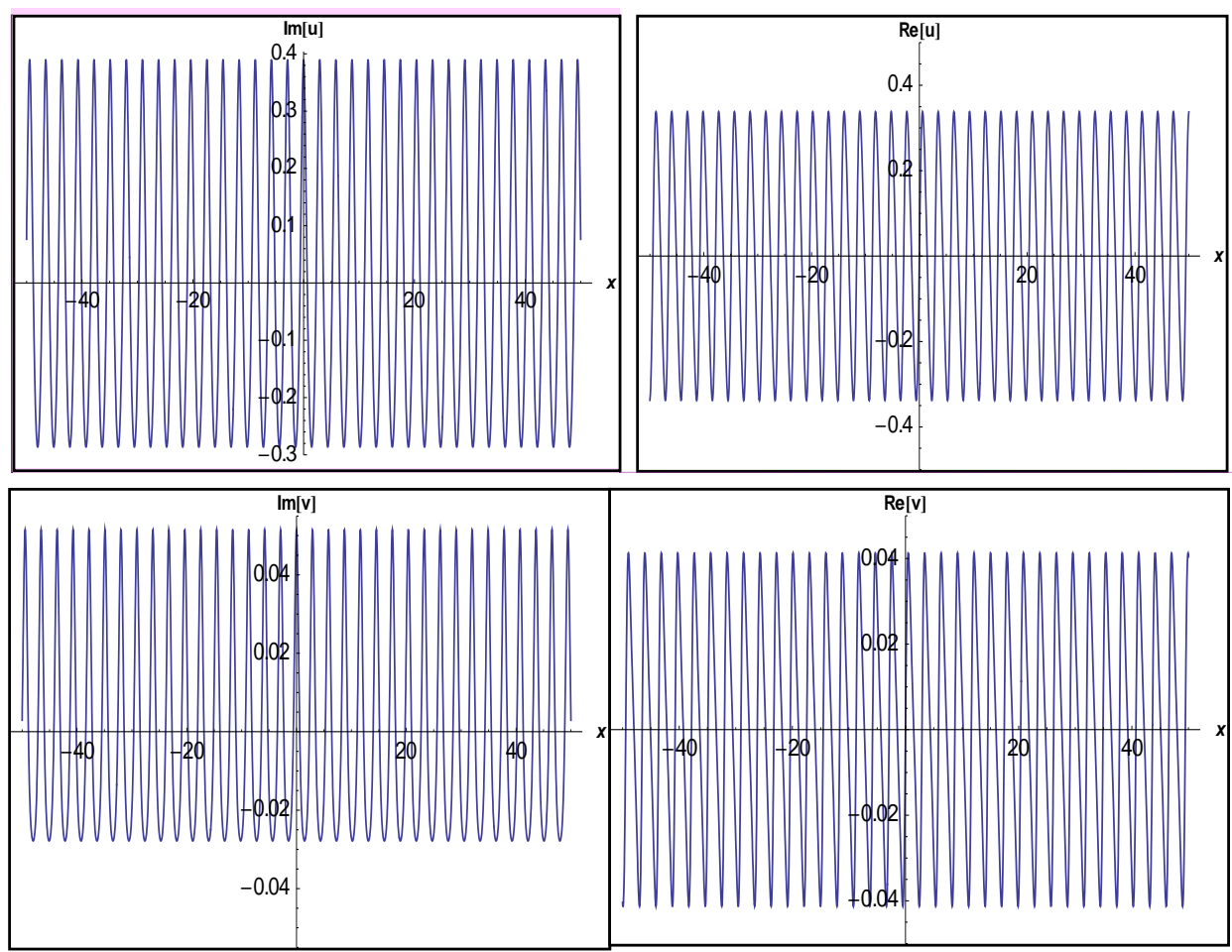

Figure 7. The $2 \mathrm{D}$ surfaces of Equation (41) by considering the values $A_{1}=2, A_{2}=3, c=5$, $\lambda=0.2, w=0.12, \varepsilon=-3, y=t=0.2,-50<x<50$. 

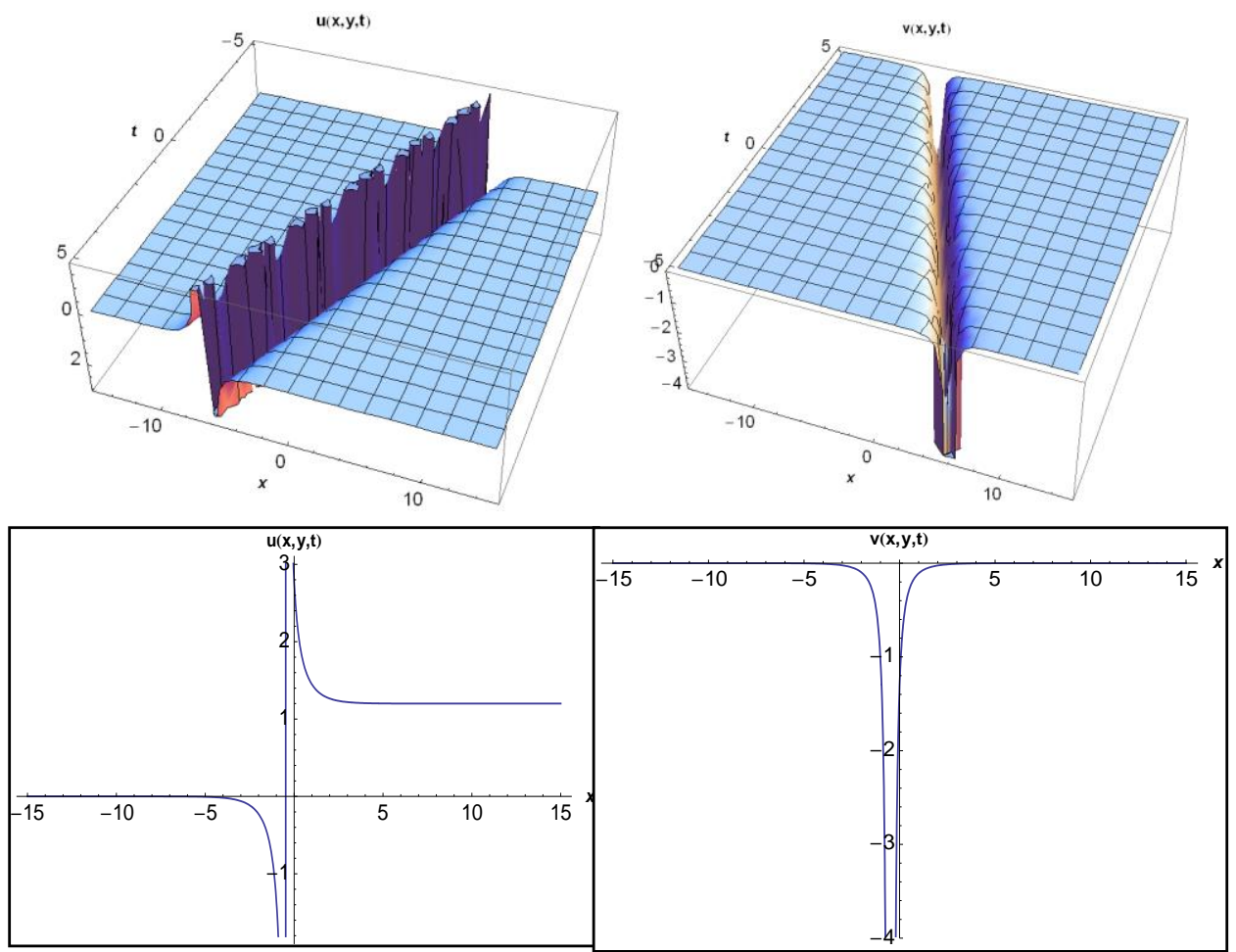

Figure 8. The $3 \mathrm{D}$ and $2 \mathrm{D}$ surfaces of Equation (42) by considering the values $A_{0}=2, B_{0}=3, k=5$, $\lambda=0.2, w=6, \varepsilon=4, y=0.2,-50<x<50,-50<t<50$, and $t=0.2$,for $2 \mathrm{D}$ surfaces.
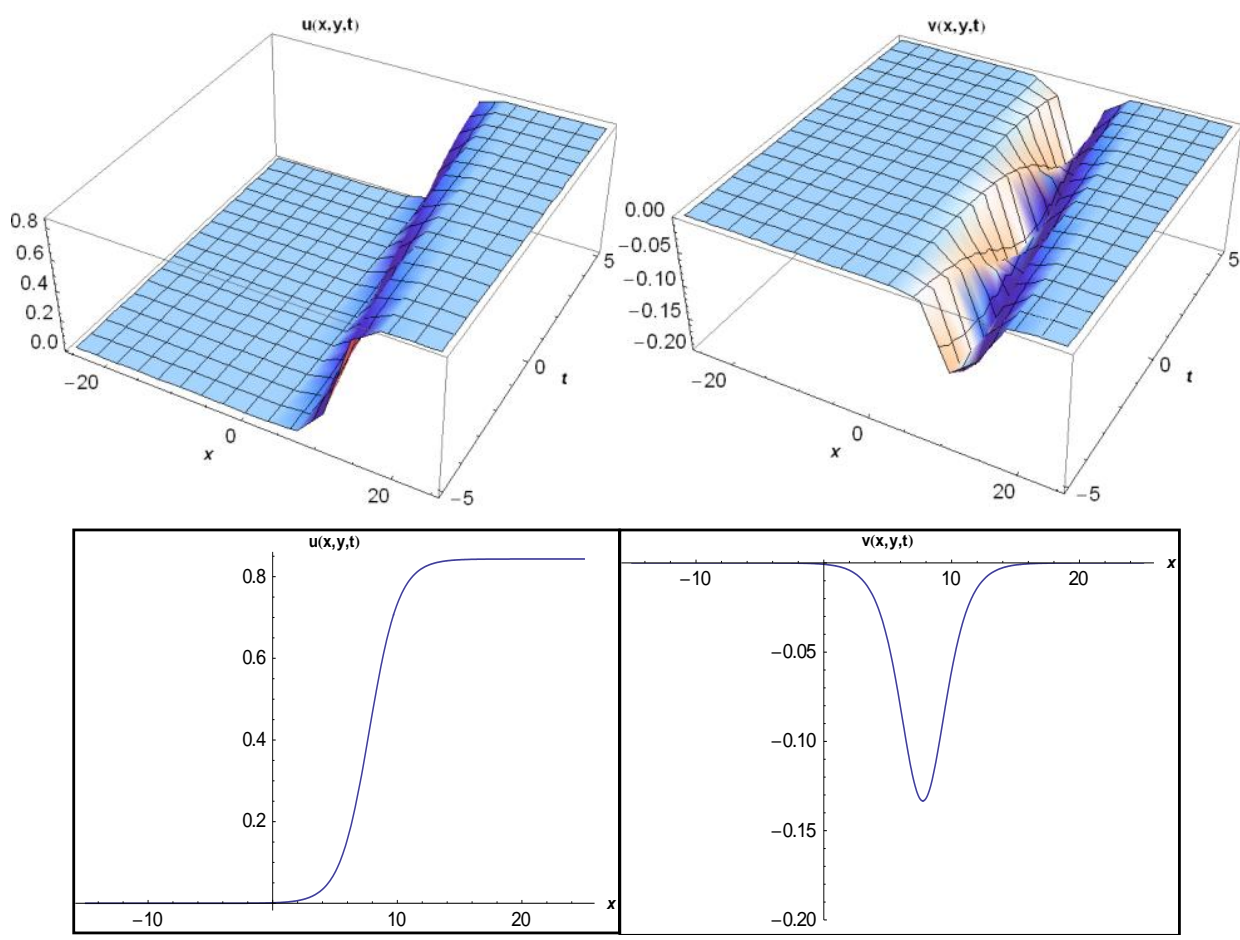

Figure 9. The $3 \mathrm{D}$ and $2 \mathrm{D}$ surfaces of Equation (47) by considering the values $A_{3}=-2, B_{2}=3, \lambda=2$, $\mu=0.6, w=0.5, \varepsilon=4, y=0.2,-25<x<25,-5<t<5$, and $t=0.2$,for $2 \mathrm{D}$ surfaces. 

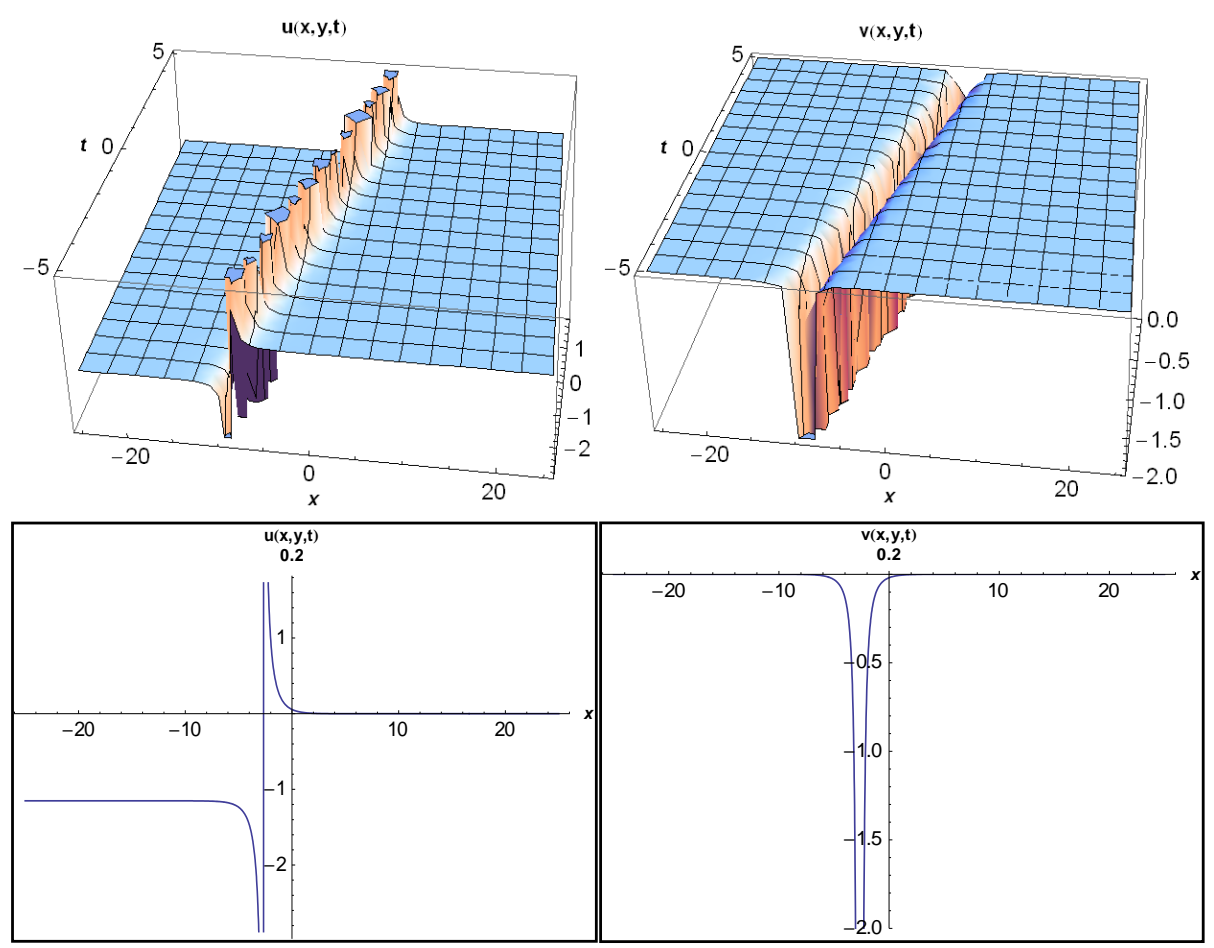

Figure 10. The $3 \mathrm{D}$ and $2 \mathrm{D}$ surfaces of the Equation (48) by considering the values $c=2, \lambda=0.2$, $\mu=-0.1, w=0.5, \varepsilon=4, y=0.2,-25<x<25,-5<t<5$, and $t=0.2$,for $2 \mathrm{D}$ surfaces.
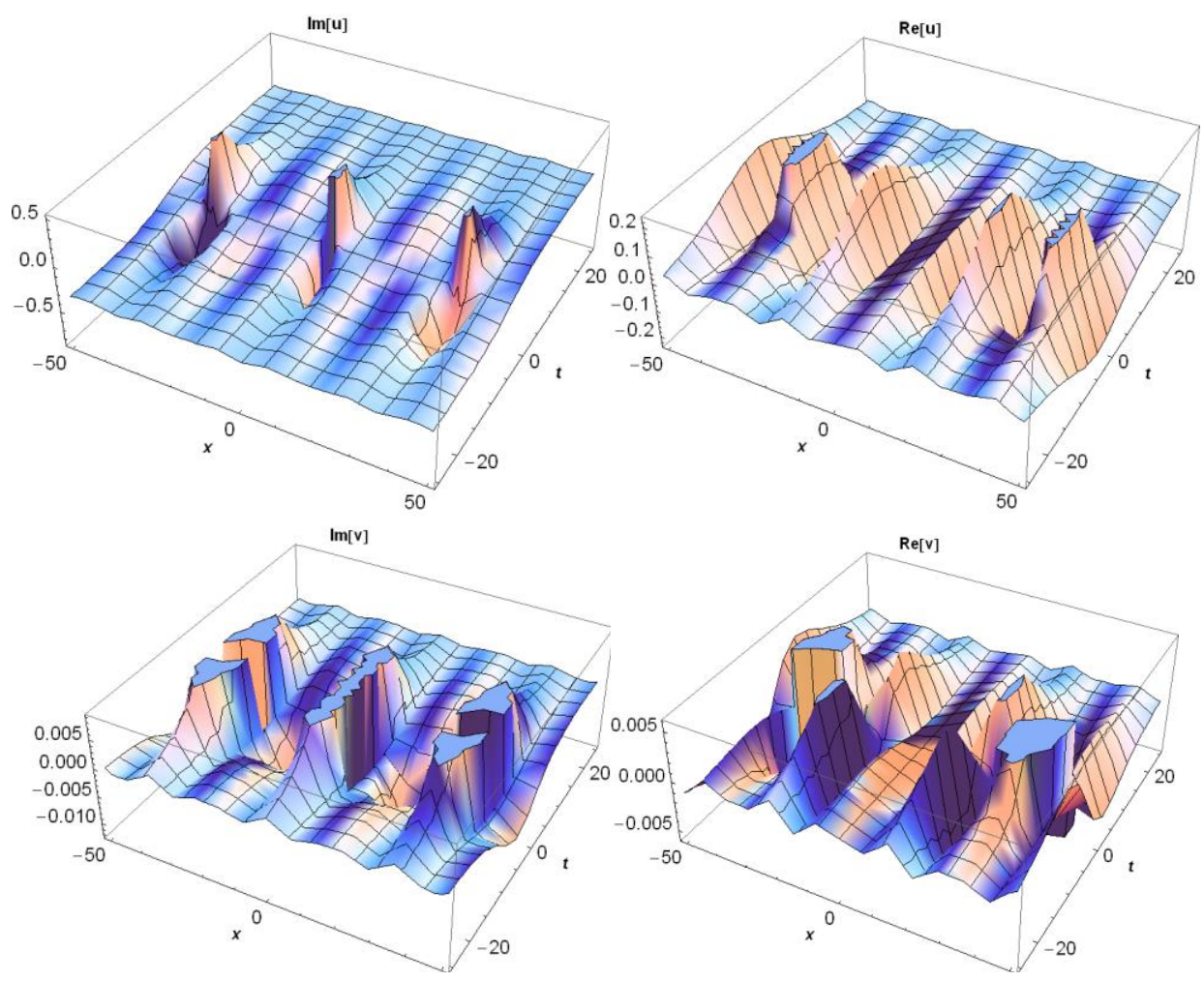

Figure 11. The 3D surfaces of Equation (57) by considering the values $b=0.2, c=0.3, d=0.4, w=0.12$, $\varepsilon=3, y=0.2,-50<x<50,-25<t<25$. 

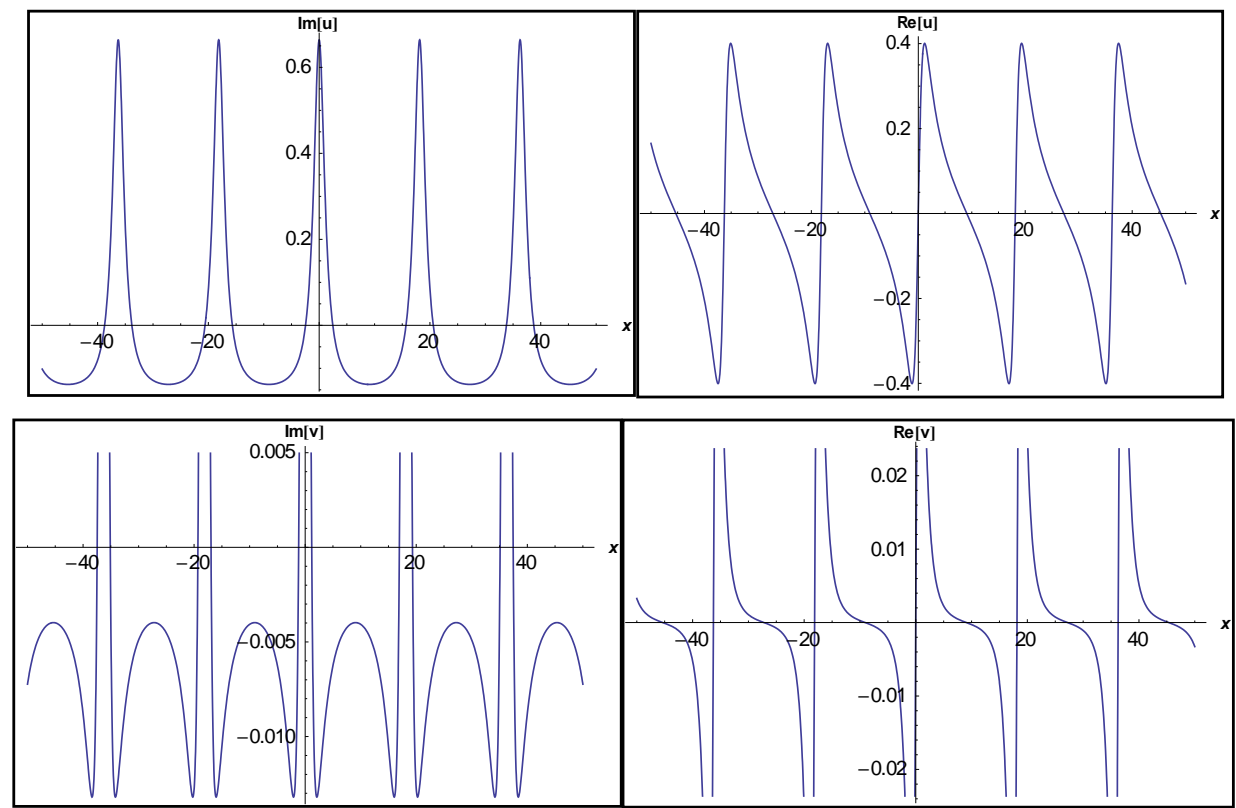

Figure 12. The 2D surfaces of Equation (57) by considering the values $b=0.2, c=0.3, d=0.4, w=0.12$, $\varepsilon=3, y=t=0.2,-50<x<50$.
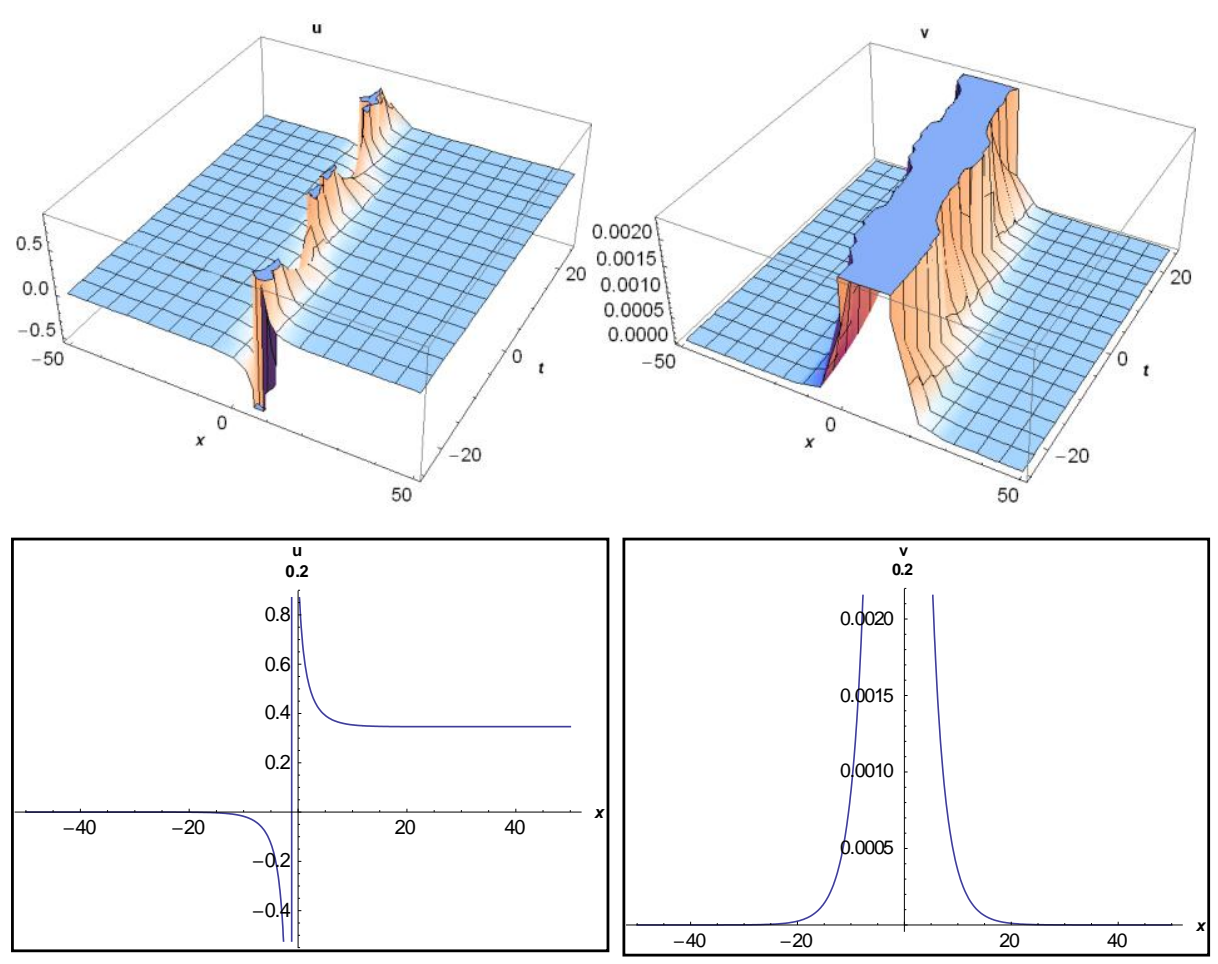

Figure 13. The $3 \mathrm{D}$ and $2 \mathrm{D}$ surfaces of Equation (58) by considering the values $b=0.2, c=0.3$, $d=0.4, w=0.12, \varepsilon=3, y=0.2,-50<x<50,-25<t<25$, and $t=0.2$, for 2D surfaces. 

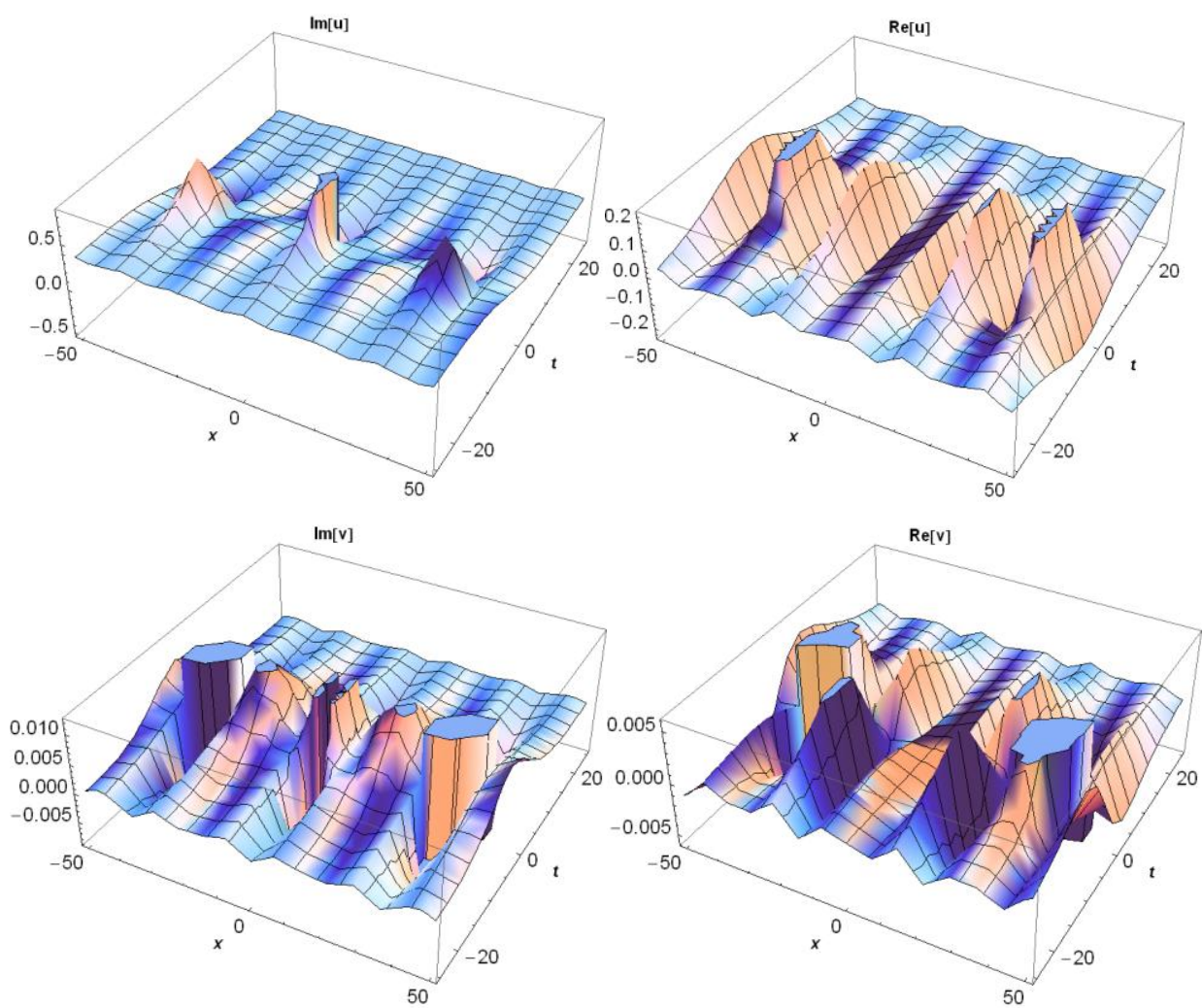

Figure 14. The 3D surfaces of Equation (59) by considering the values $b=0.2, c=0.3, d=0.4, w=0.12$, $\varepsilon=3, y=0.2,-50<x<50,-25<t<25$.
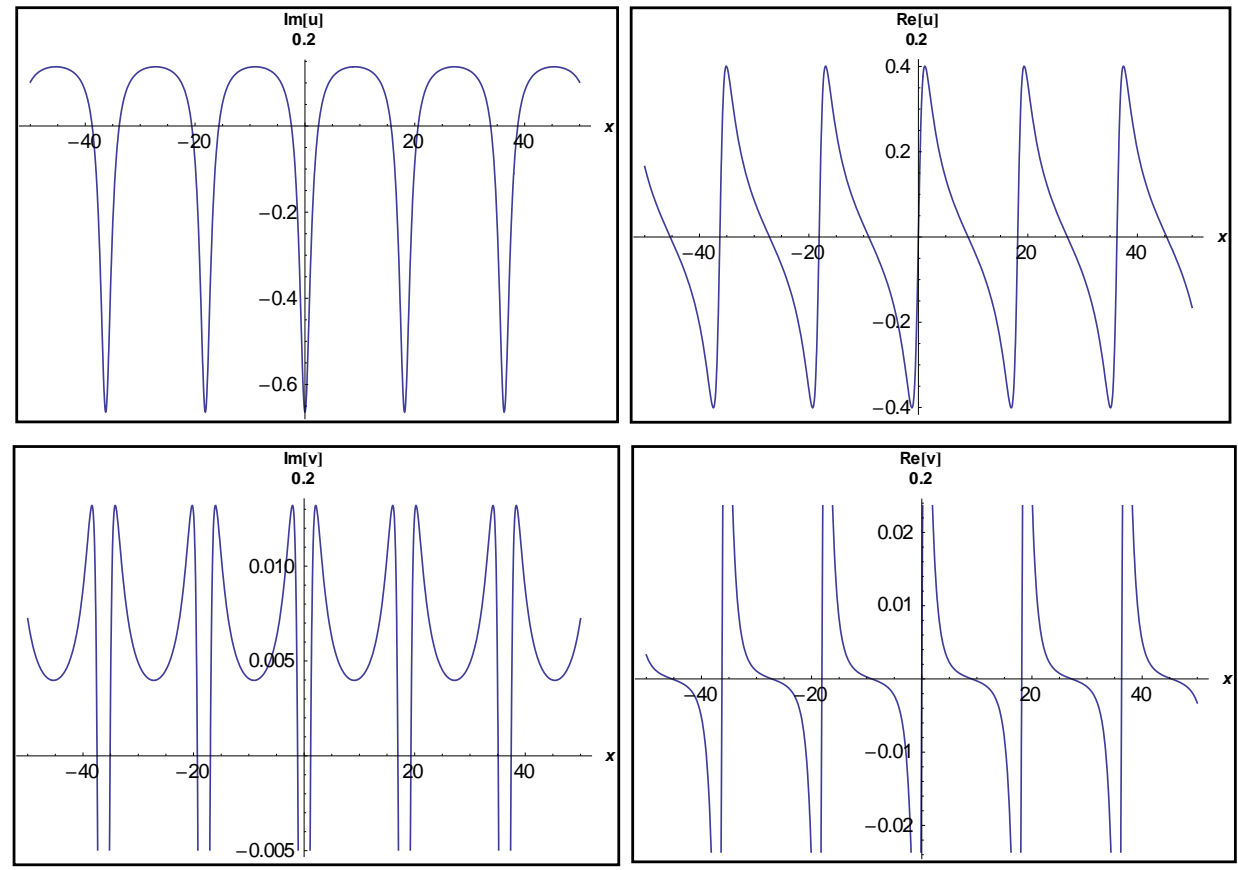

Figure 15. The 2D surfaces of Equation (59) by considering the values $b=0.2, c=0.3, d=0.4, w=0.12$, $\varepsilon=3, y=t=0.2,-50<x<50$. 
Acknowledgments: The authors would like to thank the reviewers for their valuable comments and suggestions to improve the present work.

Author Contributions: All authors have equally contributed to this paper. They have read and approved the final version of the manuscript.

Conflicts of Interest: The authors declare no conflict of interest.

\section{References}

1. Cho, Y.-S.; Sohn, D.-H.; Oh Lee, S. Practical modified scheme of linear shallow-water equations for distant propagation of tsunamis. Ocean Eng. 2007, 34, 1769-1777. [CrossRef]

2. Rodrigues, H.S.; Monteiro, M.T.T.; Torres, D.F.M. Dynamics of Dengue epidemics when using optimal control. Math. Comput. Model. 2010, 52, 1667-1673. [CrossRef]

3. Silva, C.J.; Torres, D.F.M. Modeling TB-HIV syndemic and treatment. J. Appl. Math. 2014. [CrossRef]

4. Mul, O.V.; Torres, D.F.M.; Kravchenko, V.P. Dynamics of controlled hybrid systems of aerial cable-ways. Nonlinear Anal. Hybrid Syst. 2008, 2, 431-440. [CrossRef]

5. Qiu, Z. Dynamical behavior of a vector-host epidemic model with demographic structure. Comput. Math. Appl. 2008, 56, 3118-3129. [CrossRef]

6. Zhang, T.; Gou, Q. Traveling Wave Solutions for Epidemic Cholera Model with Disease-Related Death. Sci. World J. 2014. [CrossRef] [PubMed]

7. Mari, L.; Bertuzzo, E.; Righetto, L.; Casagrandi, R.; Gatto, M.; Rodriguez-Iturbe, I.; Rinaldo, A. Modelling cholera epidemics: The role of waterways, human mobility and sanitation. J. R. Soc. Interface 2012, 9, 376-388. [CrossRef] [PubMed]

8. Bekir, A.; Güner, Ö.; Bilgil, H. Optical soliton solutions for the variable coefficient modified Kawahara equation. OptikInt. J. Light Electron Opt. 2015. [CrossRef]

9. Baskonus, H.M.; Bulut, H. On some new analytical solutions for the (2+1)-dimensional burgers equation and the special type of dodd-bullough- mikhailov equation. J. Appl. Anal. Comput. 2015, 5, 613-625.

10. Baskonus, H.M.; Bulut, H. new hyperbolic function solutions for some nonlinear partial differential equation arising in mathematical physics. Entropy 2015, 17, 4255-4270. [CrossRef]

11. Sakkaravarth, K.; Kanna, T.; Vijayajayanthi, M.; Lakshmanan, M. Multi component long-wave and short-wave resonance interaction system: Bright solitons, energy-sharing collisions, and resonant solitons. Phys. Rev. E 2014, 90. Article ID 052912.

12. Zabolotskii, A.A. Inverse scattering transform for the Yajima-Oikawa equations with nonvanishing boundary conditions. Phys. Rev. A 2009, 80. [CrossRef]

13. Khater, A.H.; Hassan, M.M.; Callebaut, D.K. Travelling wave solutions to some important equations of mathematical physics. Rep. Math. Phys. 2010, 66, 1-19. [CrossRef]

14. Roshid, H.O.; Kabir, M.R.; Bhowmik, R.C.; Datta, B.K. Investigation of Solitary wave solutions for Vakhnenko-Parkes equation via exp-function and Exp-expansion method. SpringerPlus 2014. [CrossRef]

15. Roshid, H.O.; Rahman, N.; Akbar, M.A. Traveling waves solutions of nonlinear Klein Gordon equation by extended-expasion method. Ann. Pure Appl. Math. 2013, 3, 10-16.

16. Wazwaz, A.-M. Multiple soliton solutions and rational solutions for the (2+1)-dimensional dispersive long water-wave system. Ocean Eng. 2013, 60, 95-98. [CrossRef]

17. Paquin, G.; Winternitz, P. Group theoretical analysis of dispersive long wave equations in two space dimensions. Physica D 1990, 46, 122-138. [CrossRef]

18. Chun-Long, Z.; Jian-Ping, F.; Li-Qun, C. Localized excitations with and without propagating properties in (2+1)-dimensions obtained by a mapping approach. Chin. Phys. 2005, 14, 676-682. [CrossRef]

19. Fei, J.-X.; Zheng, C.-L. Localized excitations in a dispersive long water-wave system via an extended projective approach. Z. Naturforschung A 2007, 62, 140-146. [CrossRef]

20. Hafez, M.G.; Alam, M.N.; Akbar, M.A. Travelling wave solutions for some important coupled nonlinear physical models via the coupled Higgs equation and the Maccari system. J. King Saud Univ. Sci. 2015, 27, 105-112. [CrossRef]

21. Uddin, S.; Alam, N.; Hossain, S.M.S.; Samiu, H.; Akbar, M.A. Some new exact traveling wave solutions to the (3+1)-dimensional zakharov-kuznetsov equation and the burgers equations via exp ( $-\phi)$-expansion method. Front. Math. Appl. 2014, 1, 1-8. 
22. Roshid, H.O.; Rahman, M.A. The $\exp (-\Phi(\eta))$-expansion method with application in the (1+1)-dimensional classical Boussinesq equations. Results Phys. 2014, 4, 150-155. [CrossRef]

23. Abdelrahman, M.A.E.; Zahran, E.H.M.; Khater, M.M.A. The $\exp (-\phi(\xi))$-Expansion Method and Its Application for Solving Nonlinear Evolution Equations Mahmoud. Int. J. Mod. Nonlinear Theory Appl. 2015, 4, 37-47. [CrossRef]

24. Hafez, M.G.; Alam, M.N.; Akbar, M.A. Application of the $\exp (-\Phi(\eta))$-expansion Method to Find Exact Solutions for the Solitary Wave Equation in an Unmagnatized Dusty Plasma. World Appl. Sci. J. 2014, 32, 2150-2155.

25. Zheng, B. Application of a generalized Bernoulli sub-ODE method for finding traveling solutions of some nonlinear equations. WSEAS Trans. Math. 2012, 7, 618-626.

(c) 2016 by the authors; licensee MDPI, Basel, Switzerland. This article is an open access article distributed under the terms and conditions of the Creative Commons by Attribution (CC-BY) license (http://creativecommons.org/licenses/by/4.0/). 